Sie enthält in $100 \mathrm{~g}$ das Lösliche aus $0,5 \mathrm{~g}$ Opium; annähernd 0,05 g Morphin.

Vorsichtig aufzubewahren.

Spez, Gewicht 0,897 bis 0,902 .

\title{
Tinctura Pimpinellae.
}

$\mathrm{Zu}$ bereiten aus

\author{
Bibernelltinktur.
}

Einem Teile Bibernellwurzel . . . . . . . . . . 1 mit

Fünf Teilen verdünnten Weingeistes . . . . . . 5

Eine Tinktur von bräunlichgelber Farbe, vom Geruche der Wurzel und widerlichem, kratzendem Geschmacke.

Spez. Gewicht 0,905 bis 0,910 .

\section{Tinctura Ratanhiae.}

$\mathrm{Zu}$ bereiten aus

Ratanhiatinktur.

Einem Teile Ratanhiawurzel . . . . . . . . . . 1

mit

Fünf Teilen verdünnten Weingeistes . . . . . . . 5

Eine Tinktur von dunkelweinroter Farbe, in der Verdünnung himbeerrot, ohne Geruch, von stark zusammenziehendem, herbem Geschmacke.

Spez. Gewicht 0,913 bis 0,918 .

Witteilung aus dem pharmacentischen Institut der Oniversitat Stralsburg.

\section{Beiträge zur Kenntnis der Bukublätter.}

Von Dr. Y. S him o y a ma, z. Z. in Tokio (Japan).

Die Bukublätter, welche sich in früheren Zeiten eines gewissen medizinischen Ansehens erfreuten 1) und noch heute in England und Amerika Anwendung finden, ${ }^{2}$ ) stammen von mehreren Arten des Genus Barosma und von dem nahe verwandten Empleurum serrulatum. Die

1) Flü ckiger and Ha nbary, Pharmacographia, London 1879, 108.

2) In Deutschland 1825 durch Jobst zuerst eingeführt. F.A.F. 
genannten stidafrikanischen, besonders im Kaplande einheimischen Sträucher gehören zu der Unterfamilie Diosmeae und zeichnen sich durch das eigentlimliche Verhalten der Frucht aus. Ihr Endokarp nämlich löst sich bei der Reife elastisch vom Epikarp ab, was bei den ubrigen Gruppen der Rutaceae nicht der Fall ist. Die gewöhnlich im Handel vorkommende Droge besteht besonders aus den Blăttern der Barosma betulina, Bartl., B. crenulata, Hook., B. crenata, Kze., und B. serratifolia, Willd. 1), weniger häufig auch aus den Blättern des Empleurum serrulatum, Ait. Die Blätter der drei ersteren Sträucher stellen die breite, diejenigen der zwei letzteren die schmale Sorte vor. Sie unterscheiden sich aufserlich etwa folgendermalsen:

a) Barosma betulina, Bartl. Diese Blätter sind bis $1,4 \mathrm{~cm}$ lang, 0,8 bis $1,2 \mathrm{~cm}$ breit, meist kurz gestielt, verkehrt eiförmig, an der Spitze gestutzt, mehr oder weniger zurückgerollt, am Rande unregelmălsig doppeit gesägt.

b) B. crenulata, Hook. Bis $2,5 \mathrm{~cm}$ lang und bis $1 \mathrm{~cm}$ breit, kurz gestielt, ei-lanzettförmig, fein gesägt.

c) B. crenata, Kze. Oval eitörmig, kurz gestielt, bis $1,8 \mathrm{~cm}$ lang, $0,5 \mathrm{~cm}$ breit, stumpf, fein gekerbt.

d) B. serratifolia, Willd. Blätter bis $1,5 \mathrm{~cm}$ lang und bis $0,4 \mathrm{~cm}$ breit, lanzettlich, kurz gestielt, stumpflich und am Rande gesägt.

e) Empleurum serrulatum, Ait. Diese Blätter unterscheiden sich namentlich durch ihre beträchtliche Länge und durch hellere Farbung von den ubrigen. Sie sind bis $4 \mathrm{~cm}$ lang und bis $4 \mathrm{~mm}$ breit, schmal lanzettlich, gespitzt und am Rande fein entfernt gesägt.

Alle diese Blätter zeigen auf der unteren Fläche in der Einbuchtung der Zähne je einen grölseren Ölraum und im Gewebe der Blattspreite selbst zahlreiche, unregelmälsig vertheilte Ölrăume, welche im durchfallenden Lichte sehr leicht kenntlich sind.

Der Bau der Bukublätter ist von Fluckiger (Schweiz. Wochenschrift für Pharmacie, Dezember 1873, p. 435) untersucht worden.?) Auf dessen Wunsch unternahm ich ein erneutes Studium dieser Blätter, dessen Ergebnis in folgendem mitgeteilt werden möge.

1) Seltener (nach $\mathrm{H}$ olm es) yon B. ericoides. F.A.F.

2) Oudemans, Aanteekeningen, Rotterdam 1854-1856, p. 548, hat bereits die Schleimschicht der Bukublätter kurz erwähnt. $F . A . F$. 
Unter der aus tafelformigen Zellen bestehenden und auf der Aussen-seite mit Cuticula bekleideten Epidermis liegen schleimgebende Zellen, welche im Alkohol beobachtet eine farblose, homogene Schicht bilden, von deren Ursprung unten die Rede sein wird. Nach innen folgen alsdann zwel Lagen Palissaden, von denen die obere aus längeren, dicht gedrängten, die untere aus kürzeren und locker neben einander gereihten Zellen besteht. Die unteren Palissadenzellen stofsen mit ihrem rundlichen Ende an wenig verzweigte, chlorophyllärmere Schwammparenchymzellen.

Innerhalb der mit Spaltzffnungen ausgestatteten Epidermis der Rückseite (Unterseite) der Bukublätter findet sich ebenfalls eine Schicht lose verbundener Palissadenzellen. Doch scheint das Auftreten der Palissadenschichten auf den beiden Blattseiten nicht konstant zu sein, da ich bei manchen Blättern der gleichen Sorte nur auf der Oberseite eine einschichtige Palissadenschicht beobachtete; möglich, dafs die Blätter letzterer Art von einer anderen Species herrihren. $\mathrm{Da}$, wo Spaltöffnungen vorhanden sind, zeigt die Palissadenschicht gewöhnlich an-

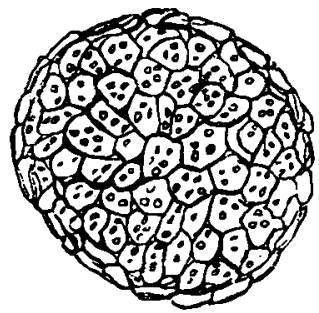

Fig. 1.

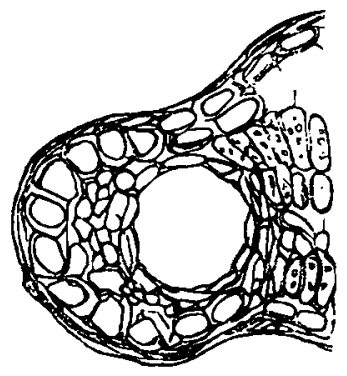

Fig. 2. Fertiger Öhraum. sehnliche Intercellularräume (Atmungshöhlen) und steht ihrerseits mit dem Schwammparenchym im Zusammenhang. Dicht. innerhalb der Epidermis der Blattunterseite sind lysigene Ölrhume eingebettet. 1) Fig. 1 ist ein jüngerer Ölraum vor der Auflösung der Zellwände und Fig. 2 ein in der Einbuchtung der Sägezähne befindlicher Ölraum in fertigen Zustande.

Sowohl in den Palissadenzellen wie im Schwammparenchym sind Drusen von Calciumoxalat eingestreut.

Unter der Epidermis der Blattoberseite, in der Hauptrippenregion, liegt die eben

1) Vergl. Flückiger und Tschirch, Grundlagen der Pharmakognosie, Berlin 1885, 219. 
erwähnte schleimgebende Schicht (Fig. 3 s), gefolgt von einer Palissadenschicht (p). Auf der Blattunterseite befindet sich in der gleichen Region unter der Epidermis Hypoderma $\left.{ }^{1}\right)(m)$, welches aus ziemlich dickwandigen, unregelmässig rundlichen Zellen besteht. Die Mitte nimmt ein Fibrovasalstrang ein, welcher aus einem scharf begrenzten, radial angeordneten $\mathrm{Xylem}$ teile (g) und aus einem wenig ausgeprägten $\mathrm{Phloëm}$ teile (b) gebildet wird. Dieses Gefaifsbündelsystem ist auf der oberen und unteren Seite von unverholzten Sk l ere n ch y m fasern (k) gestützt. Auf der oberen Seite ist diese Scheide 4-5 Zellen mächtig, während auf die untere nur 2-3 Zellenreihen kommen.

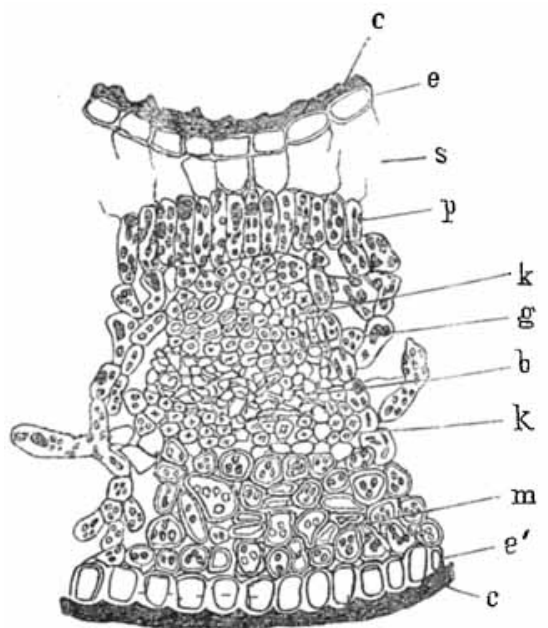

Fig. 3. Querschnitt durch ein Blatt. c Caticnle, B Bohleimschicht, p Peliasaden, 1 Sklorotische Fasern, 8 Xylem, b Phlosm, m Hypoderma, o' Epldermis der Unterseite.

Die Blattrippen der zweiten

Ordnung sind gleich beschaffen, doch sind ihre einzelnen Elemente auf eine geringere Anzabl herabgesetzt.

Bemerkenswert ist die Ablagerung von Krystallen in den Epidermiszellen. Bringt man Blatter von Barosma betulina in Alkohol und beobachtet nach einigen Tagen die blorsgelegte Epidermis, so sieht man in den unregelmărsig vieleckigen, dicht anschliersenden Zellen teils federartig, teils kugelformig angeordnete Krystalle, welche einigermalsen an die Sphärokrystalle des Inulins erinnern (Fig. 4) und sich als Hesperidin erwiesen. Sie

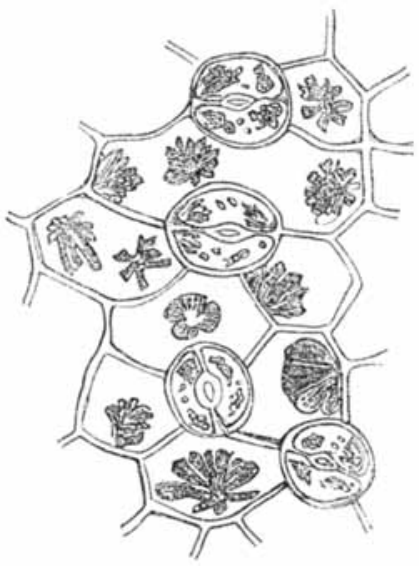

Fig. 4. Hesperidin.

1) Flückiger und Tschirch, l. c. 154. 
sind unlöslich in Wasser und Alkohol, leicht löslich in Essigsäure und Kalilauge, in letzterer mit gelblicher Farbe.

Hesperidinkrystalle findet man auch in den Schleimzellen eingelagert. Lärst man einem Querschnitte eines Blattes von B. betulina unter Alkohol vorsichtig Wasser zufliersen, so quellen die Schleimzellen auf und richten sich senkrecht zur Blattfläche empor, wobei die darin enthaltenen Gruppen des Hesperidins fadenartig auseinanderrücken. Weniger auffallend sind die Ablagerungen des Hesperidins in der Mittelschicht des Blattes; hier zeigen sie sich dem Chlorophyll ähnlich gefärbt. Es kam mir vor, als seien die Blätter von Barosma crenata und Empleurum serrulatum weniger reich an Hesperidin als die Blätter der anderen oben genannten Arten Barosma.

An den lebenden Blättern von B. alba vermilste ich die Hesperidinkrystalle gänzlich.

Zur Untersuchung der Schleimschicht dienten mir frische Blätter von Barosma alba, die Herr Professor de Bary gtitigst zur Verfügung stellte.

Diese Blätter sind höchstens $1,5 \mathrm{~cm}$ lang und $1,5 \mathrm{~mm}$ breit, spitz lanzettförmig, am Rande fein gesägt.

Betrachtet man einen Querschnitt durch die Basis (den jüngsten Teil) eines jungen Blattes, so zeigt sich unter der einzelligen Eni-

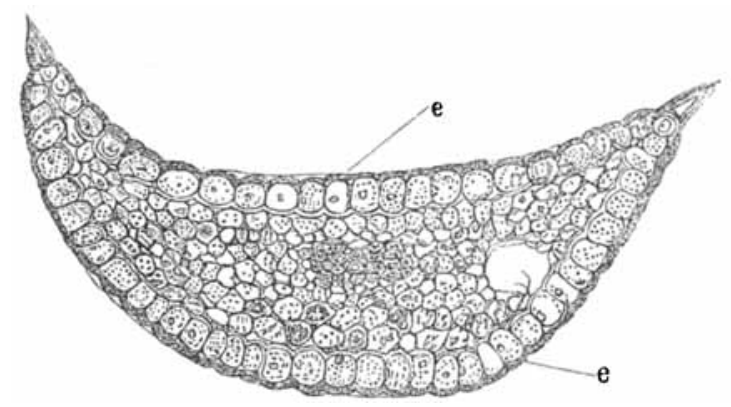

Fig. 5. Querschnitt durch das Blatt von Barosma alba. o Epidermis.

dermis (Fig. 5e) keine quellbare, schleimgebende Schicht. Bei der weiteren Entwickelung des Blattes strecken sich die Epidermiszellen radial, und in jeder Epidermiszelle tritt eine Cellulosescheidewand (Fig. 6 a) auf, so dais aus einer Epidermiszelle zwei Zellen entstehen, von denen die der Peripherie zugewendete die Funktion der Epidermis- 
zelle übernimmt (e, Fig. 6, 7 und 8). Diese Zellbildung findet aber nicht im Sinne der Zellteilung statt; wenigstens waren in den oberen Zellen keine Zellkerne aufzufinden. Später erfolgt innerlalb der Scheidewand Schleimablagerung successiv von unten nach oben und nun geht die obere Zelle in eine Schleimzelle (s, Fig. 6, 7 und 8) uber. Im jungeren Zustande wird die Membran der Schleimzelle durch Chlorzinkjodlösung

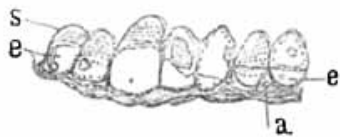

Fig. 6. Blatt von Barosma alba. a Scheiderrand, Epidermis, a Bchloimschloht, in der Bildung begrifien.

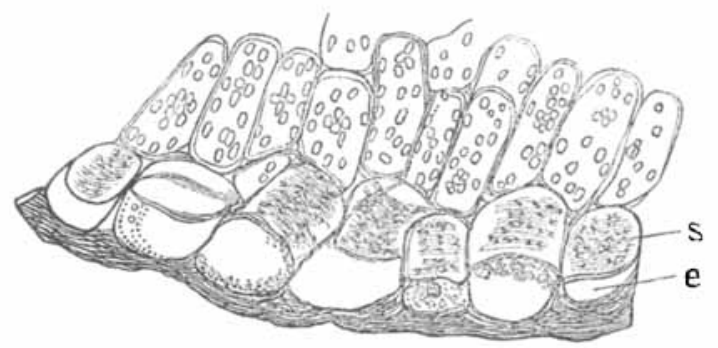

Fig. 7. Blatt von Barosma alba.

- Epidermis, B Schjeimschicht.

blau gefärbt (Cellulosereaktion); später ist dieses nicht mehr der Fall.

In s, Fig. 6 und 7, ist die Entwickelung, in Fig. 8 der fertige Zustand der Schleimschicht dargestellt. Die Schleimzellen zeigen, sowohl im jüngeren wie im ausgebildeten Zustande boobachtet, in

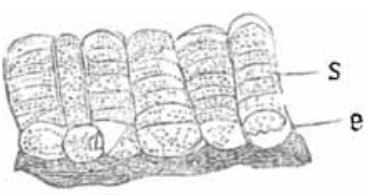

Fig. 8. Blatt von B. alba. a Epidermie, s Schleimechicht. alkoholischer Jodlösung, wie in Fig. 7 und 8 abgebildet, in einer farblosen Grundmasse eine körnige Ablagerung, die durch Jod blau gefärbt wird. Diese Masse löst sich auf Zusatz von Wasser mit bläulicher Farbe auf, wobei die Schleimzellen aufquellen.1)

Obwohl ich nicht Gelegenheit hatte, die Entstehung der Schleimzellen in anderen Barosmablättern zu verfolgen, so darf doch wohl an-

1) Herr Aschan hat aus $100 \mathrm{~g}$ lufttrockener Bukublätter $3,4 \mathrm{~g}$ bei $100^{\circ}$ getrockneten Schleim (Asche 3,7 Proz.) erhalten und daraus mittels Salzsäure rechtsdrehenden reducierenden Zucker (Traubenzucker) dargestellt. Doch scheint der Schleim nicht ein einheitlicher Körper zu sein. 
genommen werden, dafs der geschilderte Vorgang auch fir letztere gilt. In diesen sehen die Schleimzellen gleich aus wie in Barosma alba.

Radlkofer will nicht eine schleingebende Schicht der Bukublätter anerkennen, sondern erklärt die stark verdickte innere Wandung der Epidermiszellen als Herd der Schleimbildung. (Monographie der Sapindaceen-Gattung Serjania, München 1875, p. 105.) Im Gegensatze hierzu mufs ich den bezüglichen Anschauungen und Abbildungen F'lückiger's im wesentlichen beipflichten.

Die übrigen oben angeführten Bukublätter sind im ganzen anatomisch gleich gebaut, zeigen aber dGch von Art zu Art gewisse Eigentilmlichkeiten.

Innerhalb der obern Blattfläche fehlen die Schleimzellen nirgends, wohl aber bisweilen ganz oder stellenweise in den Geweben der Rückseite (Unterseite) des Blattes. So z. B. besitzen die Blätter von Barosma alba auf der Unterseite Schleimzellen, jedoch sind sie da, wo gewöhnlich Ölräume auftreten, nur wenig entwickelt. Bei Empleuram serrulatam wie bei Barosma crenata sind Schleimzellen in der untern Blattseite zu beiden Seiten der Hauptrippenregion, allerdings in geringer Entwickelung, vorhanden. Ebenso verhält es sich auch mit den Palissadenzellen. Barosma alba besitzt dergleichen beiderseits; die Unterseite zeigt sogar zwei oder drei Palissadenschichten an der Hauptrippenregion, doch fehlen sie ganz an den Stellen, wo gewöhnlich ÖIräume vorhanden sind. Ferner sieht man bei Empleurum serrulatum im Gewebe der beiden Blattseiten einschichtige Palissadenzellen.

Der Hauptblattrippenregion fehlt ausnahmsweise in Barosma alba das Hypoderma (m, Fig. 3); es ist hier durch jene Palissadenschichten vertreten. Ferner ist das Gefälsbündelsystem der Hauptrippe, welches sonst gleich beschaffen ist wie bei den anderen Barosma-Blättern, von einem einschichtigen Ring aus ziemlich stark verdickten Zellen umschlossen, welche einen bräunlichen, in Kali löslichen Inhalt führen.

\title{
Derzeitige Verhältnisse der Kaliwerke.
}

\author{
Von Dr. Emil Pfeiffer, Jena. ${ }^{\text {) }}$
}

Ein Blick auf die Gebirgskarte überzeugt uns, dafs die vom Böhmer Wald ausgehende, durch den Thüringer Wald und Teutoburger

1) Verschiedenes hier Berührte entstammt dem Handbuch der KaliIndustrie, Braunschweig 1887, vom nämlichen Verfasser. 